Tropical Journal of Pharmaceutical Research, February 2010; 9 (1): 59-66

(C) Pharmacotherapy Group,

Faculty of Pharmacy, University of Benin

Benin City, 300001 Nigeria.

All rights reserved.

Research Article

Available online at http://www.tjpr.org

\title{
Gastroretentive Floating Microspheres of Silymarin: Preparation and In Vitro Evaluation
}

\section{Rajeev Garg and GD Gupta*}

Department of Pharmaceutics and Pharmaceutical Technology, ASBASJSM College of Pharmacy, Bela, Ropar140111, India

\begin{abstract}
Purpose: To prepare and evaluate floating microspheres of silymarin for prolonged gastric residence time and increased drug bioavailability.

Methods: Cellulose microspheres - formulated with hydroxylpropyl methylcellulose (HPMC) and ethyl cellulose (EC) - and Eudragit microspheres - formulated with Eudragit ${ }^{\Theta} S 100$ (ES) and Eudragit ${ }^{\Theta} R L$ (ERL) - were prepared by an emulsion-solvent evaporation method. The floating microspheres were evaluated for flow properties based on parameters such as angle of repose and compressibility index, as well as for various other physicochemical properties including particle size, incorporation efficiency, in vitro floatability, and in vitro drug release. The shape and surface morphology of the microspheres were characterised by optical and scanning electron microscopy.

Results: Mean particle size increased while drug release rate decreased with increasing EC and ES contents of cellulose and Eudragit microspheres, respectively. Scanning electron microscopy showed pores on the surface and interior of the microspheres. The microspheres exhibited prolonged drug release for $12 \mathrm{~h}$ while still remained buoyant. Drug release kinetics, evaluated using the linear regression method, followed Higuchi kinetics and drug release mechanism was of the non-Fickian type. Conclusion: The developed floating microspheres of silymarin exhibited prolonged drug release in simulated gastric fluid for at least $12 \mathrm{~h}$, and, therefore, could potentially improve the bioavailability of the drug as well as patient compliance.
\end{abstract}

Keywords: Gastroretentive; Prolonged release; Silymarin; Floating microspheres; Ethyl cellulose; Hydroxypropyl methyl cellulose; Eudragit 


\section{INTRODUCTION}

Oral sustained drug delivery may be complicated by limited gastric residence time. Rapid gastrointestinal transit can prevent complete drug release in the absorption zone and reduce the efficacy of administered dose since the majority of drugs are absorbed in the stomach or the upper part of the small intestine.

Dosage forms that can be retained in the stomach are called gastroretentive drug delivery systems (GRDDS) [1]. Gastroretentive floating drug delivery systems (GRFDDS) have a bulk density lower than that of gastric fluids and thus remains buoyant in the stomach without affecting gastric emptying rate for a prolonged period of time [2]. While the system is floating on gastric contents, the drug is released slowly at a desired rate from the system. Both single and multiple unit systems have been developed. The single unit floating system is more popular but has the disadvantage that its purpose would not be achieved if it fails to float, or is rapidly emptied from the stomach since there is high variability of gastrointestinal transit time [3]. On the other hand, a floating system made up of multiple units may be better suited because they are claimed to reduce intersubject variability in absorption and also lower the probability of dose dumping [4].

Silymarin is a standardised seed extract which is rich in a type of flavonoid compounds known as flavonolignans [5]. The main flavonolignans in silymarin are the isomers, silybin (also known as silibinin), silydianin, and silychristin. Silymarin acts as an antioxidant, scavenger and regulator of the intracellular content of glutathione, cell membrane stabiliser and permeability regulator to prevent hepatotoxic agents from entering hepatocytes. It also acts as a promoter of ribosomal RNA synthesis [6], stimulator of liver regeneration and inhibitor of the transformation of stellate hepatocytes into myofibroblasts - the process responsible for the deposition of collagen fibres, leading to cirrhosis.

Silymarin is poorly soluble in water and, therefore, an acidic medium is essential for its dissolution. Its dose is $70-140 \mathrm{mg}$ three times a day and has low bioavailability. The low bioavailability of the drug is due to rapid biotransformation in the liver, and has a biological half-life of $6 \mathrm{~h}$. Its reatively short half-life, poor bioavailability and lipophillic nature make it a suitable candidate for gastroretentive drug delivery system.

The objective of this work was to develop and characterise gastroretentive floating microspheres of silymarin which, following oral administration, would exhibit prolonged gastric residence time and, hence increase the bioavailability of the drug.

\section{EXPERIMENTAL}

\section{Materials}

Silymarin was received as a gift from Micro Lab India. Eudragit S 100 (ES100) and Eudragit RL (ERL) were obtained from Rohm Pharma, Darmstadt, Germany while polyvinyl alcohol (PVA), hydrochloric acid $(\mathrm{HCl})$, Tween 80, hydroxylpropyl methylcellulose (HPMC) and ethyl cellulose (EC) were procured from Central Drug House, New Delhi. Dichloromethane and ethanol were purchased from E. Merck (India) Ltd, Mumbai. All the other chemicals used were of analytical grade.

\section{Preparation of microspheres}

Floating microspheres, comprising silymarin and total polymer in a 1:1 ratio, were prepared by emulsion solvent diffusion technique [7] with some modifications. The composition of the microspheres is shown in Table 1.

Trop J Pharm Res, February 2010; 9 (1): 60 
Garg \& Gupta

Table 1: Composition of floating microsphere formulations

\begin{tabular}{cccc}
\hline Code & $\begin{array}{c}\text { Polymer } \\
\text { blend }\end{array}$ & $\begin{array}{c}\text { Polymer } \\
\text { ratio }\end{array}$ & $\begin{array}{c}\text { Organic solvent ratio } \\
\text { (dichloromethane: ethanol) }\end{array}$ \\
\hline HE1 & HPMC:EC & $1: 1$ & $1: 1$ \\
HE2 & HPMC:EC & $1: 2$ & $1: 1$ \\
HE3 & HPMC:EC & $1: 3$ & $1: 1$ \\
HE4 & HPMC:EC & $1: 4$ & $1: 1$ \\
RLS1 & ERL: ES & $0: 2$ & $1: 1$ \\
RLS2 & ERL: ES & $0.5: 1.5$ & $1: 1$ \\
RLS3 & ERL: ES & $1: 1$ & $1: 1$ \\
RLS4 & ERL: ES & $1.5: 0.5$ & $1: 1$ \\
\hline
\end{tabular}

\section{Cellulose microspheres}

The polymer content was a mixture of HPMC:EC (1 g) in ratios of 1:1 (HE1), 1:2 (HE2), 1:3 (HE3) and 1:4 (HE4). The drug and polymer (in a ratio of $1: 1$ ) were dissolved in a $20 \mathrm{ml}$ mixture of dichloromethane and ethanol (1:1) at room temperature. The solution was poured slowly as a thin stream into $150 \mathrm{ml}$ of $0.01 \%$ Tween 80 maintained at $30-40{ }^{\circ} \mathrm{C}$. The emulsion was continuously stirred at a rotation speed of $300 \mathrm{rpm}$ for $1 \mathrm{~h}$ to allow the volatile solvents to evaporate. The floating microspheres were collected by decantation while the non-floating microspheres were discarded along with polymer residues. The collected microspheres were dried overnight in an oven at $40 \pm 2{ }^{0} \mathrm{C}$ and stored in a desiccator containing calcium chloride as a desiccant.

\section{Eudragit microspheres}

The polymer content of the microspheres was a mixture of $E S$ and $E R L$ in ratios of $0: 2$ (RLS1), 0.5:1.5 (RLS2), 1:1 (RLS3) and 1.5:0.5 (RLS4). The drug and polymer blend $(20 \mathrm{ml})$, in a ratio of $1: 1$, were dissolved in a mixture of dichloromethane and ethanol (1:1) at room temperature. The solution was poured slowly as a thin stream into $150 \mathrm{ml}$ of $0.2 \%$ sodium lauryl sulphate solution maintained at $30-40{ }^{\circ} \mathrm{C}$. The emulsion was continuously stirred at a rotation speed of 300 rpm for $1 \mathrm{~h}$ to allow volatile solvents to evaporate. The floating microspheres were collected by decanting, while the non-floating microspheres were discarded along with residues of the polymers. The collected microspheres were dried in an oven overnight at $40 \pm 2{ }^{\circ} \mathrm{C}$ and stored in a calcium chloride desiccator.

\section{Evaluation of silymarin and microspheres Authentication of silymarin}

Identification of silymarin was by comparison with that of an authentic sample and by verification of the presence of functional groups in its infra-red (IR) spectra. Also, various concentrations of the drug in $0.1 \mathrm{M}$ $\mathrm{HCl}$ were evaluated by ultraviolet (UV) spectroscopy (Shimadzu -1700) to determine if it would obey Beer's law.

\section{Particle size determination}

The particle size of the microspheres was determined with an optical microscope under regular polarised light, and mean particle size was calculated by measuring 100 microspheres $(n=3)$ with the help of a calibrated oculometer [8].

\section{Tapped density}

Tapping method was used to calculate tapped density. The volume of a weighed quantity of the microspheres was determined, after 100 taps, using a tapped density apparatus (Pharma Chem Machineries, model C-BD 100), as in Eq. 1. 
$\mathrm{D}_{\mathrm{T}}=\mathrm{M}_{\mathrm{T}} / \mathrm{V}_{\mathrm{T}}$

where $D_{T}=$ tapped density, $M_{T}$ is mass of microspheres and $V_{T}=$ volume of microspheres after tapping

\section{Carr's (Compressibility) index}

This parameter was calculated from bulk density (the ratio of weighed quantity of microspheres to its volume), $D_{P}$, and tapped density as in Eq 2 [9]

Compressibility index $=\left(D_{T}-D_{P}\right) / D_{T} \times 100$

\section{Angle of repose}

The angle of repose, $\theta$, of the microspheres, which measures resistance to particle flow, was determined by the fixed funnel method [10] and calculated as in Eq 3.

$\operatorname{Tan} \theta=S / D$

where $S=$ surface area of the free standing height of the microspheres heap and $D=$ diameter of the heap.

\section{Scanning electron microscopy}

Scanning electron microscopy (SEM) studies were performed to determine the porous/hollow nature of the microspheres. Surface morphology of microspheres was also noted.

\section{Drug loading}

The drug content of the floating microspheres was carried out by dissolving the microspheres in a small amount of dichloromethane in a separating funnel and extracting the drugs into $0.1 \mathrm{~N} \mathrm{HCl}$ by evaporating the dichloromethane. Determination of drug loading $(n=3)$ was carried out at $288 \mathrm{~nm}$ spectrophoto-metrically (Shimadzu 1700).

\section{In vitro floatability}

In vitro floatability studies on floating microspheres were carried out using USP XXIV dissolution apparatus II [11]. The microspheres were placed in $0.1 \mathrm{M}$ hydrochloric acid containing $0.02 \% \mathrm{v} / \mathrm{v}$ Tween 80 with the paddle rotating at $100 \mathrm{rpm}$ for $12 \mathrm{~h}$. Tween 80 served to mimic the effect of natural surfactants in the stomach. The floating and the settled portions of the microspheres were recovered separately, dried and weighed. Buoyancy (floatability) was calculated as in Eq 4.

Buoyancy $(\%)=Q_{f} /\left(Q_{f}+Q_{s}\right) \times 100$

where $Q_{f}$ and $Q_{s}$ are the weights of the floating and the settled microspheres, respectively.

\section{In vitro drug release studies}

Drug release studies were carried out in a six-basket USP XXIV dissolution apparatus type I rotating at $100 \mathrm{rpm}$ in $0.1 \mathrm{M}$ hydrochloric acid as dissolution medium (900 $\mathrm{ml}$ ) maintained at $37 \pm 0.5^{\circ} \mathrm{C}$. At specific time intervals, up to $12 \mathrm{~h}$, aliquots were withdrawn and analysed at $288 \quad \mathrm{~nm}$ spectrophotometrically (Shimadzu 1700) after suitable dilution. The withdrawn volume was replaced with an equal volume of fresh $0.1 \mathrm{M}$ hydrochloric acid to maintain sink conditions. All experiments were performed in triplicate. The drug release data were fitted to Zero order (cumulative \% drug release versus time), First order (log cumulative \% drug retained versus time) and Higuchi models (cumulative \% drug released versus square root of time) to assess the kinetics of drug release and determine the release mechanism of the drug from the floating microspheres.

\section{Statistical analysis}

Data of two sets were evaluated by paired $t$ test and one-way analysis of variance (ANOVA) were applied to check significant 
difference in drug release from different formulations. $p$ values of less than $0.05(p<$ $0.05)$ were considered to be significant.

\section{RESULTS}

\section{Silymarin authentication}

The IR spectra showed peaks at 3449.3 , 2926.8, 2366.1, 1741.3, 1083.2 and 1027.8 $\mathrm{cm}^{-1}$. which are consistent with the presence of the functional groups of silymarin. Furthermore, the calibration curve of silymarin obeyed Beer's law in the range of $20-55 \mu \mathrm{g} / \mathrm{ml}$.

\section{Physical properties of microspheres}

The mean particle size of the microspheres significantly increased $(p<0.05)$ with increasing polymer concentration. Particle size was in the range of $220.4 \pm 3.7$ to 247.6 $\pm 3.1 \mu \mathrm{m}$ for cellulose microspheres and $251.3 \pm 3.9$ to $282.7 \pm 5.2 \mu \mathrm{m}$ for Eudragit microspheres, as shown in Table 2.
Angle of repose for cellulose microspheres

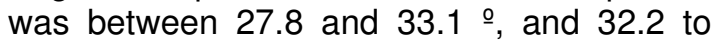
$41.3 \cong$ for Eudragit microspheres, thus indicating good flow property for both types of microspheres. The findings were supported by Carr's (compressibility) index, which was < 20 indicating good flow characteristics (Table 2).

The production yield of floating microspheres was greater than $65 \%$ for all the

formulations. In the floatation test, more than $75 \%$ of cellulose microspheres and $61 \%$ of Eudragit microspheres remained floating at the end of $12 \mathrm{~h}$ (Table 2).

The encapsulation efficiency of the prepared microspheres was in the range $85.1 \pm 2.3$ to $94.2 \pm 1.4$ for cellulose microspheres and $87.7 \pm 2.6$ to $72.8 \pm 3.7$ for Eudragit microspheres. Scanning electron microscopy revealed pores on the microsphere as well as a hollow microsphere interior (see Fig 1).

Table 2: Some characteristics of silymarin microspheres

\begin{tabular}{llcccccc}
\hline Code & $\begin{array}{l}\text { Mean } \\
\text { particle } \\
\text { size }(\mu \mathrm{m})\end{array}$ & $\begin{array}{l}\text { Bulk } \\
\text { density } \\
\left(\mathrm{g} / \mathrm{cm}^{3}\right)\end{array}$ & $\begin{array}{l}\text { Tapped } \\
\text { density } \\
\left(\mathrm{g} / \mathrm{cm}^{3}\right)\end{array}$ & $\begin{array}{l}\text { Carr's } \\
\text { index } \\
(\%)\end{array}$ & $\begin{array}{l}\text { Angle of } \\
\text { repose }\left({ }^{0}\right)\end{array}$ & $\begin{array}{l}\text { Encapsulation } \\
\text { efficiency }(\%)\end{array}$ & $\begin{array}{l}\% \\
\text { Buoyancy }\end{array}$ \\
\hline HE1 & $220.4 \pm 3.7$ & 0.582 & 0.692 & 15.89 & 27.76 & $85.1 \pm 2.3$ & 75.7 \\
HE2 & $232.8 \pm 4.5$ & 0.547 & 0.676 & 19.08 & 33.13 & $87.4 \pm 4.8$ & 82.5 \\
HE3 & $239.1 \pm 2.8$ & 0.530 & 0.678 & 18.86 & 31.40 & $88.5 \pm 5.7$ & 90.4 \\
HE4 & $247.6 \pm 3.1$ & 0.410 & 0.497 & 17.50 & 30.50 & $94.2 \pm 1.4$ & 94.1 \\
RLS1 & $282.7 \pm 5.2$ & 0.631 & 0.757 & 16.64 & 32.21 & $87.7 \pm 2.6$ & 68.1 \\
RLS2 & $272.9 \pm 4.7$ & 0.535 & 0.642 & 16.67 & 34.55 & $83.4 \pm 3.5$ & 79.8 \\
RLS3 & $261.9 \pm 3.5$ & 0.473 & 0.578 & 18.17 & 35.83 & $77.8 \pm 4.3$ & 84.4 \\
RLS4 & $251.3 \pm 3.9$ & 0.527 & 0.602 & 12.46 & 41.28 & $72.8 \pm 3.7$ & 61.2 \\
\hline
\end{tabular}

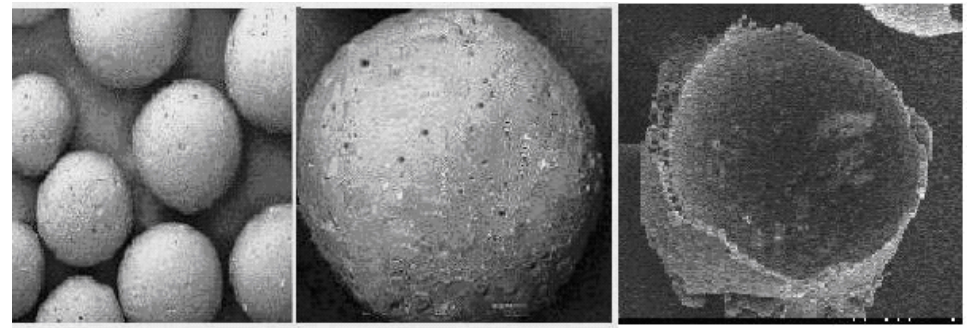

Fig 1: Typical scanning electron microphotographs (SEM) of the developed floating microspheres 
In the in vitro drug release studies, the highest cumulative drug released by the cellulose microspheres after $12 \mathrm{~h}$ was $70 \pm$ $2.7 \%$ and this was for HE1, i.e., the formulation with the lowest content of ethyl cellulose. Maximum drug released for HE2, HE3 and HE4 was $68.0 \pm 1.4,65.0 \pm 1.5$ and $58.0 \pm 2.2 \%$, respectively (see Figure $2 \mathrm{~A}$ ). For the Eudragit microspheres, the formulation with the highest content of ERL (i.e., RLS4) showed a maximum drug release of $46.2 \pm 1.7 \%$. Maximum drug release decreased to $37.5 \pm 2.2,35.731 .5$ and $32.2 \pm$ $2.1 \%$ for RLS3, RLS2 and RLS1, respectively, as Figure $2 \mathrm{~B}$.
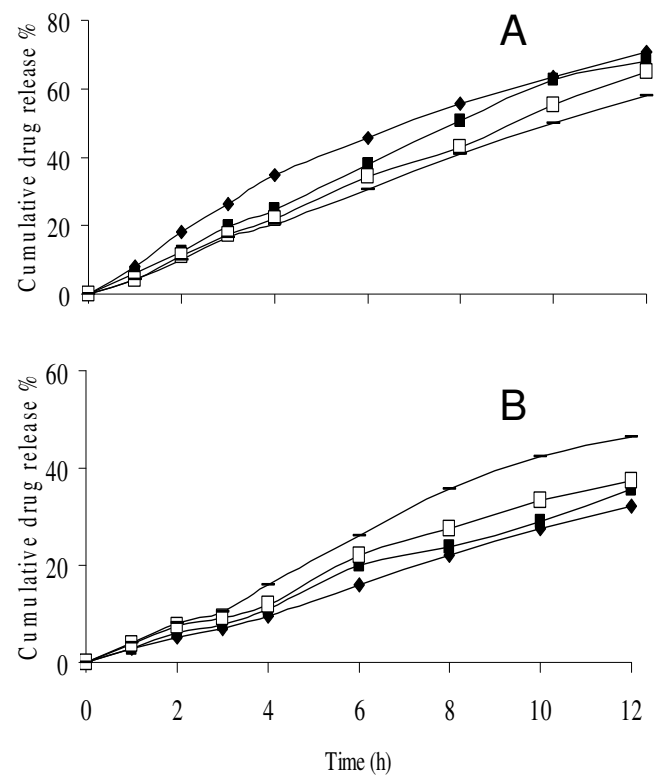

Fig 2: In vitro release profile of silymarin from cellulose floating microspheres in $0.1 \mathrm{M} \mathrm{HCl}$

Note: (A) $\bullet=$ HE1; $\cdot=$ HE2; $\square=$ HE3; - = HE4, and from Eudragit floating microspheres (B): RLS1; ' = RLS2; $\square$ = RLS3; - = RLS4

\section{DISCUSSION}

Cellulose microspheres were prepared by gradually increasing EC concentration in combination with a fixed concentration of
HPMC in order to assess the effect of polymer (especially EC) concentration on microsphere size. It would be expected that the viscosity of the polymer mixture would increase as polymer concentration rose, resulting in enhanced interfacial tension and hence, formation of larger particles. The mean particle size of Eudragit microspheres was greater than that of cellulose microspheres, and this may be viscosityrelated. All the microsphere formulations showed excellent flowability as represented by angle of repose $\left(\leq 35^{\circ}\right)$, except for RLS4 and this might be due to its high content of ERL.

Encapsulation efficiency rose with increase in EC concentration for cellulose microspheres while for Eudragit microspheres, this parameter decreased with increase in ERL content. This could be due to the higher permeability characteristics of HPMC and $E R L$ which would facilitate the diffusion of part of the entrapped drug to the surrounding medium during the preparation of the microparticles.

The SEM images in Fig 1 showed the spherical and hollow nature of the microspheres as well as the presence of pores on their surface. Their sphericity contributed considerably to their very good flow properties while their hollow nature would mean lower microsphere density. A large proportion of the microspheres -61 and $75 \%$ for Eudragit and cellulose microspheres, respectively - remained floating after $12 \mathrm{~h}$. This period was sufficient for up to $70 \%$ of cellulose microspheres (HE1) and $46 \%$ of the Eudgragit microspheres (RLS4) to be released. Thus, prolonged floatation was achieved without apparent gelation. Floatation might have been influenced by the low bulk and tapped densities of the microspheres. Microspheres with the highest levels pf ERL (RLS4) and HPMC (HE1) were least buoyant. It is likely that the surfactant incorporated in the formulations would have increased their wettability and hence, hydration, more than in 
the other microspheres which had lower levels of the permeable ERL and HPMC. Consequently, the increased amount of absorbed liquid medium replaced the air inside the floating microspheres, thus rendering them less buoyant $[12,13]$.

Silymarin release from cellulose microspheres decreased with increasing EC concentration. Increase in the content of EC (which is less permeable than HPMC) would increase polymer matrix density and thus result in increased diffusional pathlength, leading to a decrease in drug release from the microsphere. Another factor might be that the smaller microspheres formed at low EC concentration had a larger surface area exposed to the dissolution medium, thus, giving rise to faster drug release.

On the other hand, the greater the content of $E R L$, the higher was the rate of drug release from Eudragit microspheres. ES is insoluble in acidic medium and also exhibits low permeability [14]. It is an anionic copolymer of methacrylic acid and methyl methacrylate containing free carboxylic and ester groups. Its very low permeability results from the high intermolecular attraction between its molecules. Hydrogen bonding between the hydroxyl groups of the carboxylic moiety and the carbonyl oxygen of ester group increases the degree of compactness of the polymer and decreases its porosity and permeability. $E R L$, however, is a copolymer of acrylic and methacrylic acid esters with a low content of quaternary ammonium groups. The ammonium groups present as salts promotes permeability and act as a channeling agent for the entrance of the liquid medium through the floating microsphere wall, causing it to swell. This facilitates the diffusion of the dissolved drug out of the microsphere into the dissolution medium. Thus, by varying the ratio of HPMC and EC in the cellulose microspheres, and ES and ERL in Eudragit microspheres, the rate of release of silymarin can be controlled.

The zero order plots of different formulation were found to be fairly linear, as indicated by their high regression values $\left(R^{2}=0.9700\right.$ 0.9984 ). Thus, it seems that drug release from the floating microspheres followed Zero order kinetics. Data based on the Higuchi model usually provide a clue to the diffusion mechanism of drug release from matrix systems such as the floating microspheres developed in this work. $R^{2}$ values based on the Higuchi model ranged from 0.9680 to 0.9991 (Table 3). As these values were close to 1.0 , the drug release mechanism of the developed floating microspheres can be said to be Higuchian and, therefore, matrix diffusion-controlled.

Table 3: Regression coefficients $\left(R^{2}\right)$ derived from release data for various formulations using different drug release models.

\begin{tabular}{clll}
\hline \multirow{2}{*}{ Code } & \multicolumn{3}{c}{$\mathbf{R}^{2}$} \\
\cline { 2 - 4 } & $\begin{array}{l}\text { Zero } \\
\text { order }\end{array}$ & $\begin{array}{l}\text { First } \\
\text { order }\end{array}$ & $\begin{array}{l}\text { Higuchi } \\
\text { kinetics }\end{array}$ \\
\hline HE1 & 0.97 & 0.9993 & 0.9991 \\
HE2 & 0.9936 & 0.990 & 0.9866 \\
HE3 & 0.9984 & 0.9839 & 0.9843 \\
HE4 & 0.9975 & 0.9941 & 0.9886 \\
RLS1 & 0.9974 & 0.9940 & 0.9680 \\
RLS2 & 0.9946 & 0.9927 & 0.9735 \\
RLS3 & 0.9908 & 0.9936 & 0.9762 \\
RLS4 & 0.9893 & 0.9912 & 0.9776 \\
\hline
\end{tabular}

\section{CONCLUSION}

We can conclude that floating microspheres of silymarin prepared with a suitable blend of $E S$ and ERL on the one hand, and HPMC and EC on the other, demonstrate satisfactory release and floating properties. The developed floating microspheres also showed good physicochemical properties. Drug release from the formulations followed Zero order kinetics and the mechanism of drug release was diffusion-controlled.

\section{REFERENCES}

1. Streubel $A$, Siepmann J, Bodmeier $R$. Gastroretentive drug delivery system. Expert Opin Drug Deliv. 2006; 3: 217-233.

2. Singh $B N$, Kim KH. Floating drug delivery systems: an approach to oral controlled drug delivery via 
Garg \& Gupta

gastric retention. J. Control. Release. 2000; 63: 235-239.

3. Whitehead L, Fell JT, Collett JH, Sharma HL, Smith AM. Floating dosage forms: An in vivo study demonstrating prolonged gastric retention. $J$ Control Release. 1998; 55: 3-12.

4. Rouge N., Leroux J C, Cole E T, Doelker E, Buri P. Prevention of the sticking tendency of floating minitablets filled into hard gelatin capsules. Eur. J. Pharm. Biopharm. 1997; 43: 165-171.

5. Muriel $P$, Mourelle M. Prevention by siymarin of membrane alterations in acute CCl4 liver damage. J Appl Toxicol. 1990; 10: 275.

6. Sonnenbichler J, Zetl I. Biochemical effects of the flavonolignan silibinin on RNA, protein and DNA synthesis in rat liver. Progr Clin Biol Res. 1986; 213: 319-31.

7. Kawashima $Y$, Niwa $T$, Takeuchi $H$, Hino $T$, Itoh $Y$. Hollow microspheres for use as a floating controlled drug delivery system in the stomach. J. Pharm. Sci. 1992; 81: 135-140.

8. Martin A. Micromeritics. Physical Pharmacy, fourth ed. Lea Feiger, Philadelphia, 1993: 431-432.
9. Lin S, Kao Y. Solid particulates of drug bcyclodextrin inclusion complexes directly prepared by a spray drying technique. Int $J$ Pharm. 1989; 56: 249-259.

10. Sinha VR, Agarwal MK, Kumaria R. Influence of formulation and excipients variables on the pellet properties prepared by extrusion spheronization. Curr. Drug Delivery. 2005; 2: 1-8.

11. The United State Pharmacopoeia XXIV, United state Pharmacopoeial Convention, Rock Ville 2000: pp 1941-1943.

12. Okor RS, Obi CE. Drug release through aqueous based film coatings of acrylate- methacrylate, a water insoluble copolymer. Int $J$ Pharm. 1990; 58: 89-91.

13. Li CL, Martini LG, Ford JL, Roberts M. The use of hypromellose in oral drug delivery. J Pharm Pharmacol. 2005; 57: 533-546.

14. Moldenhauer $M$, Nairn J. The control of ethyl cellulose microencapsulation using solubility parameters. J Control Release. 1992; 22: 205218. 